\title{
Avaliação da escrita através da fotogrametria - estudo da preensão trípode dinâmica*
}

\section{Handwriting assessment through photogrammetry - dynamic tripod grasp evaluation}

\author{
Pedro Henrique Tavares Queiroz de Almeida ${ }^{1}$, Camila Beatriz Sousa \\ Sorensen ${ }^{2}$, Luis Alberto Magna ${ }^{3}$, Daniel Marinho Cezar Cruz ${ }^{4}$, \\ Iracema Serrat Vergotti Ferrigno 5
}

http://dx.doi.org/10.11606/issn.2238-6149.v24i1p38-47

Almeida PHTQ, Sorensen CBS, Magna LA, Cruz DMC, Ferrigno ISV. Avaliação da escrita através da fotogrametria - estudo da preensão trípode dinâmica. Rev Ter Ocup Univ São Paulo. 2013 jan./abr.;24(1):38-47.

RESUMO: INTRODUÇÃO: Dentre as preensões utilizadas para a escrita a trípode dinâmica apresenta a maior prevalência entre a população. Apesar de considerada padrão para a escrita manual, são escassos estudos que envolvam a análise da amplitude articular das mãos durante esta atividade. OBJETIVO: Obter as variações das amplitudes de movimento das articulações distais do membro superior durante a atividade de escrita manual em adultos. METODOLOGIA: 50 adultos jovens, destros e sem comprometimento no membro superior tiveram suas mãos fotografadas durante a escrita e posteriormente analisadas através do Software de Avaliação Postural (SAPO).

RESULTADOS: Foi observado um padrão comum de pronação de antebraço, abdução entre I e II metacarpianos e flexão de metacarpofalangeana e interfalangeana proximal do II dedo, similar às descrições encontradas na literatura. CONCLUSÃO: Foi possível estabelecer um padrão postural por meio da análise fotogramétrica, demonstrando a aplicabilidade deste método para a análise da motora do membro superior durante a atividade de escrita manual.

DESCRITORES: Escrita manual; Terapia Ocupacional; Avaliação; Fotogrametria.
Almeida PHTQ, Sorensen CBS, Magna LA, Cruz DMC, Ferrigno ISV. Handwriting assessment through photogrammetry - dynamic tripod grasp evaluation. Rev Ter Ocup Univ São Paulo. 2013 jan./ abr.;24(1):38-47.

ABSTRACT: INTRODUCTION: Different grasp patterns can be used for handwriting and the dynamic tripod is the most prevalent among adults. Although it is considered an ideal grasp pattern, there are few studies that analyses the range of motion of hand joints during this activity. OBJECTIVE: To obtain the variations of range of motion in distal upper limb articulations during a handwriting task among healthy adults. METHODS: 50 young adults, right-handed and without impairment in the upper limb had their hands photographed during a handwriting task and analyzed with Postural Assessment Software (SAPO). RESULTS: A common pattern of forearm pronation, abduction of the first interdigital space, flexion of metecarpophalangeal and proximal interphalangeal joints of the index finger was observed among subjects, matching previous studies and descriptions for this grasp pattern. CONCLUSION: It was possible to establish a standard postural pattern for handwriting through photogrammetric analysis, demonstrating the applicability of this method for the upper limb.

KEY WORDS: Handwriting; Occupational therapy; Evaluation; Photogrammetry.

\footnotetext{
*O presente trabalho é parte do projeto de pesquisa "Estudo da Escrita Manual", desenvolvido pelo Laboratório de Análise Funcional e Ajudas Técnicas (LAFATec) do Departamento de Terapia Ocupacional da UFSCar. Os resultados parciais desta pesquisa foram apresentados no XII Congresso Brasileiro de Terapia Ocupacional e IX Congresso Latino-americano de Terapia Ocupacional, na modalidade pôster, durante os dias 11 a 14 de Outubro de 2011, em São Paulo, SP.

1. Professor Assistente - Universidade de Brasília - Curso de Terapia Ocupacional da Faculdade de Ceilândia Mestre em Terapia Ocupacional pela UFSCar.

2. Terapeuta Ocupacional - Universidade de São Carlos. Bolsista PIBIT - CNPq.

3. Professor Titular - Departamento de Genética Médica - Universidade Estadual de Campinas. Livre-Docente na Área de Genética Médica pela UNICAMP.

4. Professor Adjunto - Departamento de Terapia Ocupacional - Universidade Federal de São Carlos. Doutor em Educação Especial pela UFSCar.

5. Professora Adjunta - Departamento de Terapia Ocupacional - Universidade Federal de São Carlos. Doutora em Ciências Médicas pela UNICAMP; Coordenadora do LAFATec - DTO/UFSCar.

Endereço para correspondência: Pedro H. T. Q. de Almeida. Rua 37 Sul, Lote 08 - Ap.402 - Águas Claras, Brasília, DF. CEP: 71931 540. E-mail: pedroalmeida.to@gmail.com
} 


\section{Introdução}

medida articular é um importante dado de
avaliação em reabilitação de pessoas com
disfunção física e importante parâmetro para as pesquisas na área. Para a avaliação postural e mensuração da amplitude de movimento, inúmeros métodos tem sido empregados, dependendo dos dados a serem analisados e seu respectivo grau de complexidade ${ }^{33}$.

Tradicionalmente, terapeutas e médicos têm utilizado o goniômetro como método de mensuração articular durante a prática clínica. A goniometria exige uma posição inicial, estabilização dos eixos do instrumento sobre os segmentos articulares e acompanhamento do goniômetro até a amplitude de movimento (ADM) máxima ${ }^{8}$, tendo boa confiabilidade inter e intra avaliadores ${ }^{32}$ e ampla utilização e aceitação entre fisioterapeutas, médicos e terapeutas ocupacionais.

Com o advento dos meios digitais de comunicação, assim como o uso de novas tecnologias para captação, registro e armazenamento de dados, a fotogrametria digital tem sido considerada como método alternativo, de fácil aplicação, alta precisão e reprodutibilidade dos resultados ${ }^{11,19,31,38}$. O método consiste na obtenção de fotografia digital e sua manipulação através de softwares para a mensuração de ângulos, distâncias horizontais e verticais como modo de analisar posturas e arcos de movimento articular ${ }^{32}$.

A aplicação da fotogrametria abrange intervenções na área da saúde relacionadas a disfunções mandibulares, respiratórias e avaliações posturais ${ }^{7,17,18,24}$. São comuns a estes estudos a obtenção de altos valores de reprodutibilidade das medidas inter e intra avaliadores, sugerindo boa confiabilidade do método ${ }^{19}$. Contudo os estudos atuais envolvem apenas alguns seguimentos do corpo humano, principalmente o tronco, a face e os membros inferiores.

Como um importante segmento corporal, a mão está envolvida nas mais diversas atividades do cotidiano, expressando uma variedade de funções graças a uma complexa harmonia entre as várias estruturas que a compõe ${ }^{28}$. A completa função manual é atingida através da combinação de estímulos aferentes e eferentes, representados por feedback tátil e precisas respostas musculares ${ }^{23,46}$.

Uma das tarefas mais complexas e dinâmicas da função manual é a escrita. A transcrição de conceitos e ideias por meio de símbolos, realizada através do uso de objetos de escrita como canetas e lápis é considerada a mais elaborada forma de comunicação ${ }^{35}$.

Escrever requer não apenas uma resposta motora eficiente, mas também a integração de funções percepto- cognitivas de forma eficaz, para coordenar o movimento do lápis ou caneta em uma direção específica e alcançar o resultado esperado ${ }^{2,29}$.

Pesquisas atuais têm procurado analisar a escrita manual atentando-se para a definição de melhores padrões de preensão ${ }^{1,14,16,22,25,27,40,41}$. Preensões que envolvam maior quantidade de movimentos distais são consideradas mais eficientes para a escrita, enquanto padrões que concentrem a movimentação sobre estruturas proximais tem sido descritos como inadequados, apresentando limitações na velocidade e precisão dos movimentos ${ }^{21}$, podendo levar a dificuldades no desempenho adequado da escrita ${ }^{13}$, ocasionando reduzida legibilidade ${ }^{44}$, dor e fadiga nos membros superiores ${ }^{34}$.

Estudos de comparação entre o desempenho funcional de indivíduos que utilizam diversos tipos de preensão são escassos e não existem estudos que reconheçam um tipo de preensão como mais eficaz. Observa-se, entretanto, que a preensão trípode dinâmica é a mais prevalente entre a população infantil ${ }^{37}$, adolescente, adulta ${ }^{41}$ e idosa ${ }^{42}$.

Neste padrão preensor o manuseio do objeto de escrita é realizado através dos dedos I, II e III, que seguram e movimentam o objeto de escrita (Figura 1). A caneta é posicionada entre o I e II dedo, sendo apoiada na borda radial da falange média ou distal do III dedo ${ }^{34}$. O primeiro espaço interdigital é mantido aberto, com os dedos IV e V flexionados, sustentando o arco longitudinal da mão ${ }^{37}$.

Os movimentos necessários para a escrita são concentrados nas articulações distais do membro superior, sobretudo metacarpofalangeanas e interfalangeanas dos dedos I e II $^{12}$.

Grande parte dos estudos sobre a escrita manual utiliza observações clínicas ${ }^{6,36,37}$, associadas a medidas goniométricas tradicionais $^{40,43}$ e análise cinemática ${ }^{4,9,10,30}$ como ferramenta metodológica, inexistindo estudos que apresentem dados para fundamentar a classificação de padrões de preensão por meio da fotogrametria.

\section{Objetivos}

O presente estudo teve como objetivo a análise fotogramétrica do padrão preensor trípode dinâmico de jovens universitários, durante uma atividade de escrita, como meio de obter as variações das amplitudes de movimento articular para fornecer dados normativos que embasem a avaliação do padrão motor dos membros superiores durante a atividade de escrita manual.

\section{Metodologia}

A pesquisa foi delineada como um estudo 
observacional transversal e foi aprovada pelo parecer $n^{\circ} 322 / 2010$ do Comitê de Ética em Pesquisa em Seres Humanos da Universidade Federal de São Carlos (CEP/
UFSCar) - CAAE (Certificado de Apresentação para Apreciação Ética) no 0017.0.135.000-10.

A coleta de dados foi realizada entre os meses de janeiro a julho de 2011.

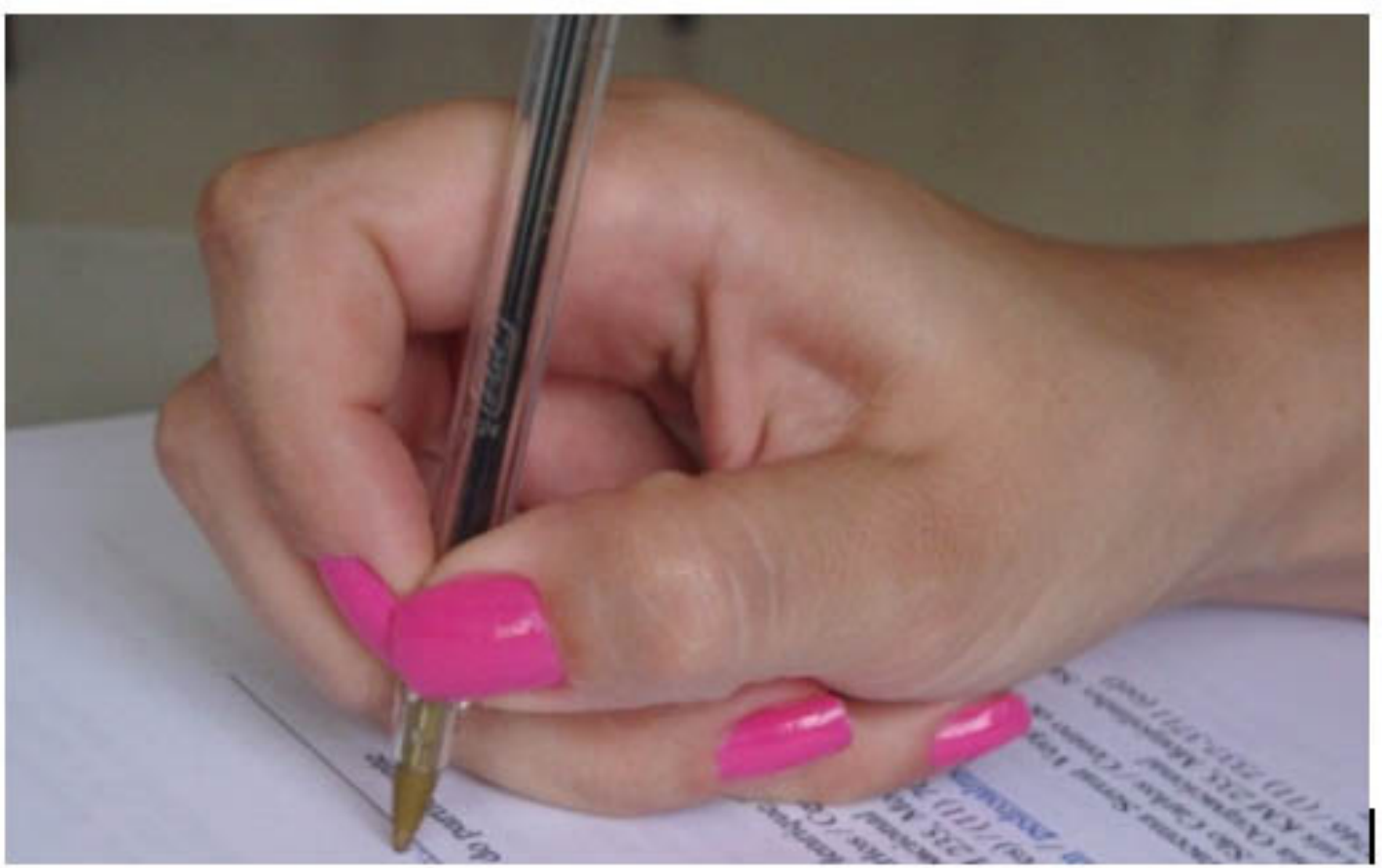

Figura 1 -Exemplo de Preensão Trípode Dinâmica. Observa-se a preensão da caneta entre os dedos I e II, com apoio desta sobre o dedo III. O I dedo encontra-se em oposição ao II dedo

\section{Casuística}

Participaram da pesquisa estudantes universitários saudáveis, de 18 a 30 anos, destros, que utilizavam a preensão trípode dinâmica como forma dominante durante a escrita manual. Foram excluídos indivíduos com histórico de fadiga, dor e desconforto, com sinais ou diagnóstico de doença, trauma ou sequelas que pudessem interferir no desempenho sensório-motor dos membros superiores.

Foram convidados a participar da pesquisa estudantes dos cursos de graduação e pós-graduação da Universidade Federal de São Carlos, por meio de folders, mensagens enviadas por e-mail institucional e comunicação veiculada no endereço eletrônico da universidade.

A classificação do tipo de preensão dos participantes foi realizada através da análise de fotos e filmagens baseada em descrições conceituais de padrões de preensão feitas por estudos de análise motora da escrita ${ }^{2,12,37,41,43}$.
Participaram da pesquisa 50 estudantes universitários (20 do sexo masculino e 30 do sexo feminino), destros, selecionados aleatoriamente a partir de uma amostra inicial de 288 indivíduos que tiveram seu padrão de preensão classificado previamente como trípode dinâmico.

\section{Ambiente de Coleta de Dados}

A pesquisa foi realizada no Laboratório de Análise Funcional e Ajudas Técnicas - LAFATEC, do Departamento de Terapia Ocupacional da UFSCar, ambiente com iluminação, ruído e temperatura controlados; a preparação do ambiente procurou minimizar aspectos que alterassem a qualidade da escrita, modificando a demanda e atenção dos participantes e consequentemente modificando seu desempenho.

\section{Material}

Foi utilizada uma câmera fotográfica digital modelo 
DSC-W35, de 7,2 megapixels de resolução de imagem, do fabricante SONY, computador desktop, marca TOPIS, com processador Intel Core Duo 2,80 GHz, 2GB de memória RAM e HD de 320gb, monitor Samsung T220 LCD 22 polegadas e teclado padrão ABNT-2 e mouse óptico, ambos da marca BRIGHT.

Os participantes foram posicionados sentados em uma cadeira universitária com prancheta de madeira fixa à direita, assento e encosto estofados, modelo CF30 do fabricante Móveis Office, utilizaram folhas de papel almaço com distância de pauta de $13 \mathrm{~mm}$, do fabricante Tilibra S.A. e caneta esferográfica, modelo BIC Cristal ${ }^{\circledR}$, azul com espessura média de ponto de $1.0 \mathrm{~mm}$.

Para análise fotogramétrica das imagens foi utilizado o Software de Avaliação Postural (SAPO) v. 0.61, desenvolvido por Ferreira et al. (2010) com apoio da Fundação de Amparo à Pesquisa do Estado de São Paulo (FAPESP) e acesso livre, disponibilizado pelo site http://sapo.incubadora.fapesp.br/ portal.
O SAPO é um programa computacional para fins acadêmicos ou clínicos ${ }^{15}$, disponível em domínio público, funcionando através da digitalização de pontos anatômicos demarcados, que possibilita funções tais como a calibração da imagem, medição de distâncias e principalmente a mensuração de ângulos corporais.

\section{Método}

Foram selecionados pontos anatômicos localizados sobre proeminências ósseas para facilitar o posicionamento dos marcadores digitais do software e realizar as medições da ADM de cada articulação. A escolha dos pontos se baseou em critérios e recomendações utilizados para a avaliação goniométrica dos membros superiores, normatizados e descritos pela Sociedade Brasileira de Terapeutas da Mão como referência para a obtenção de tais medidas ${ }^{26}$. Os pontos anatômicos selecionados estão descritos na Tabela 1.

Tabela 1 - Pontos anatômicos utilizados como marcadores para avaliação fotogramétrica

\begin{tabular}{|c|c|}
\hline Região & Ponto Selecionado \\
\hline Antebraço & $\begin{array}{l}\text { Metade do comprimento da distância entre o epicôndilo lateral e a cabeça da ulna, } \\
\text { projetado entre o rádio e a ulna; face posterior }\end{array}$ \\
\hline Antebraço & $1 / 3$ distal do comprimento do rádio; face lateral \\
\hline Antebraço & Tubérculo de Lister \\
\hline Punho & Articulação Trapeziometacarpiana do I dedo; face lateral \\
\hline Metacarpos & Cabeças dos metacarpos II, III e V; face posterior \\
\hline Dedo I & Articulação metacarpofalangeana do polegar; face posterior \\
\hline Dedo I & Articulação interfalangeana do polegar; face posterior \\
\hline Dedo II & Articulação metacarpofalangeana do II dedo; face lateral \\
\hline Dedo II & Articulação interfalangeana proximal do II dedo; face lateral \\
\hline Dedo II & Articulação interfalangeana distal do II dedo; face lateral \\
\hline
\end{tabular}

Os indivíduos foram orientados a sentarem de forma confortável, posicionar o papel e segurar a caneta da meneira como estavam habituados. Cada indivíduo foi orientado a escrever uma frase simples, padronizada e composta por dez caracteres - "a vida é bela".

A escolha da frase procurou evitar o uso de recursos visuais ou auditivos pelo indivíduo, e consequentemente evitar modificações no desempenho, priorizando uma escrita fluida ${ }^{5,39}$.

Durante a atividade quatro fotografias do membro superior direito foram captadas, pela mesma pesquisadora, nos planos lateral direito, lateral esquerdo, frontal e superior. A distância entre a câmera e a folha de papel foi padronizada em $70 \mathrm{~cm}$ e a posição da câmera foi mantida através do uso de um tripé com altura de um metro a partir do solo para os planos laterais e frontal e $1,5 \mathrm{~m}$ para o plano superior.

Posteriormente, as imagens, calibradas em um zoom padrão de $50 \%$, foram inseridas no programa SAPO e três examinadores, alunos do último ano do curso 
de graduação em Terapia Ocupacional e previamente treinados procederam à análise fotogramétrica das articulações do antebraço, punho, metacarpofalangeanas e interfalangeanas dos dedos I e II (Figura 2).

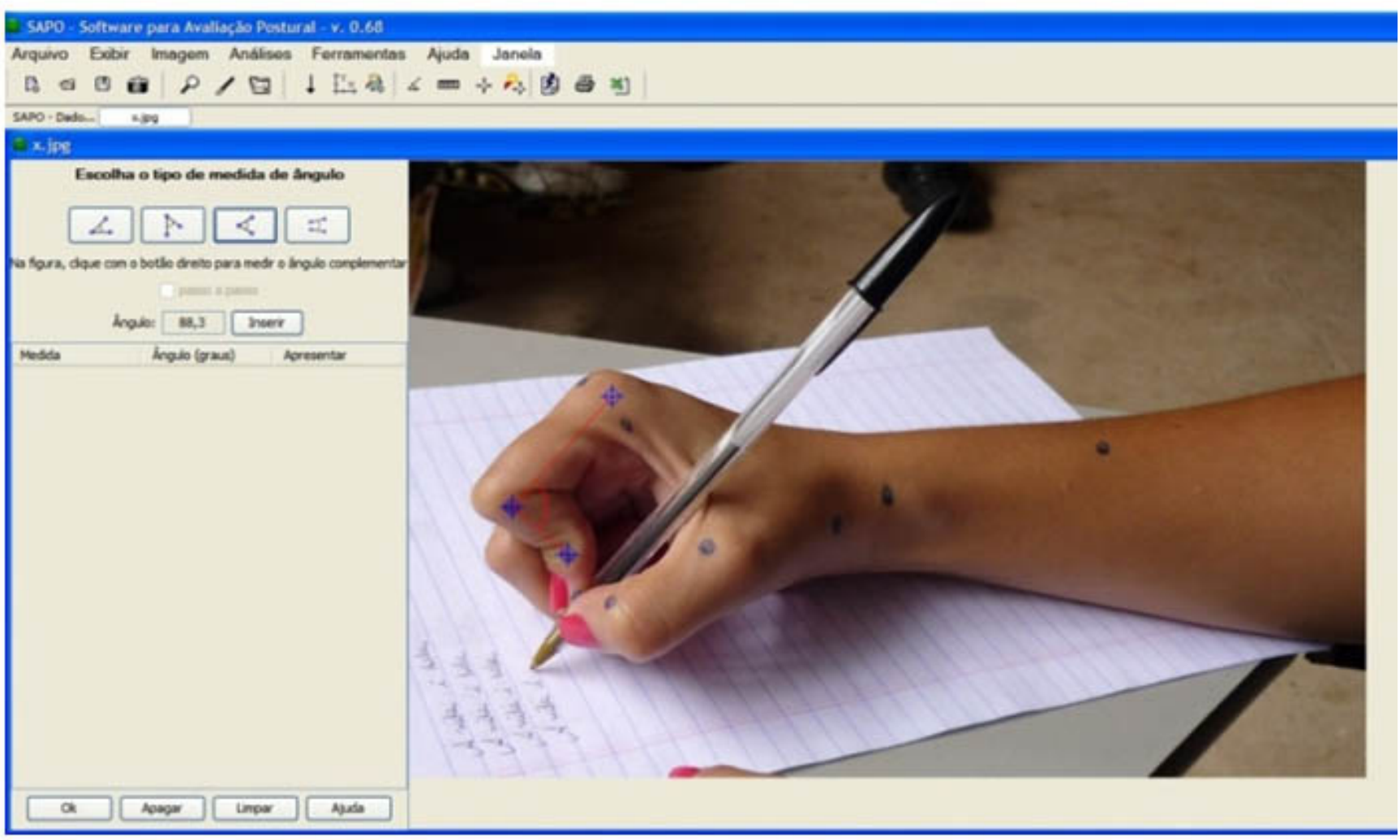

Figura 2 - Exemplo de Análise Fotogramétrica através do programa SAPO - a ADM da articulação interfalangeana proximal é mensurada durante a escrita por meio da preensão Trípode Dinâmica

As mensurações obtidas foram armazenadas em planilha do software Microsoft Excel 2007 e para cada ângulo descrito obteve-se a média aritmética das medições feitas pelos três avaliadores. Esses dados foram analisados para verificar o grau de variabilidade angular de cada articulação, considerando como aceitável um desviopadrão de 5 (cinco) pontos percentuais inter-avaliadores.

\section{Resultados}

Variação da ADM

Através da observação das imagens e análise fotogramétrica, definiu-se a prevalência postural adotada, observando-se que a maior parte dos indivíduos realizou a atividade em pronação do antebraço, desvio radial, abdução do primeiro espaço interdigital, flexão das articulações metacarpofalangeana (MF) e interfalangeanas proximal (IFP). As posturas adotadas pelas interfalangeanas distais (IFD) e demais articulações durante a atividade de escrita são ilustradas no Gráfico 1.

A variação da amplitude de movimento articular dos sujeitos durante a escrita manual, do tipo trípode dinâmica se apresenta conforme a Tabela 2. 
Gráfico 1 - Prevalência do padrão postural do membro superior, durante a escrita manual de adultos saudáveis que utilizam a preensão trípode dinâmica

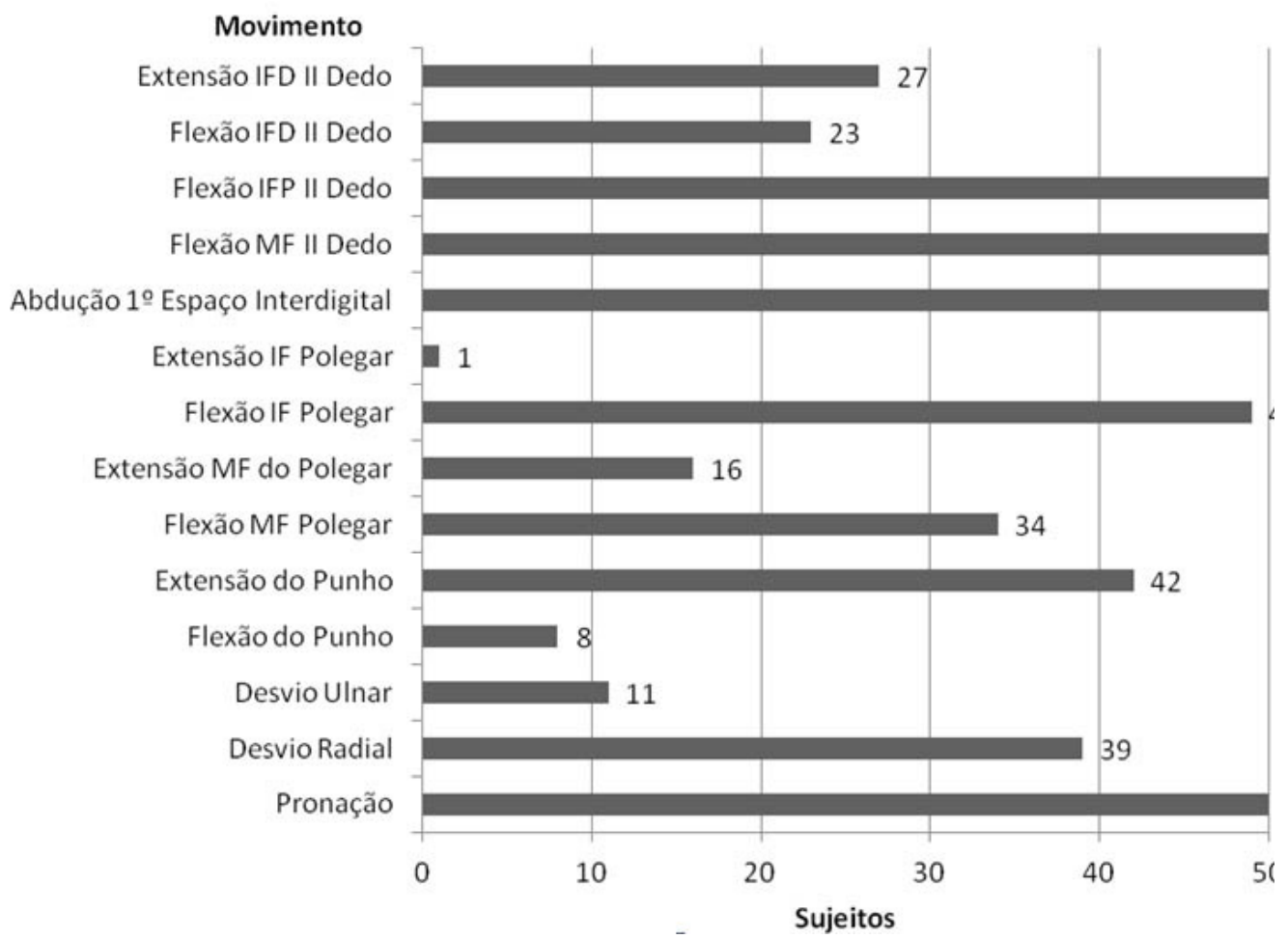

Tabela 2 - Amplitudes articulares médias, desvio padrão e variação mínima e máxima da ADM observada entre os sujeitos durante atividade de escrita manual

\begin{tabular}{lll}
\hline Movimento & Média (Desvio Padrão) & Variacão ADM \\
Pronação de Antebraço & $25,09^{\circ}( \pm 1,03)$ & $9,7^{\circ}$ a $43,8^{\circ}$ \\
Desvio Radial do Punho & $10,04^{\circ}( \pm 1,36)$ & $0,3^{\circ}$ a $26,3^{\circ}$ \\
Desvio Ulnar do Punho & $5,10^{\circ}( \pm 1,53)$ & $0,56^{\circ}$ a $10,9^{\circ}$ \\
Flexão do Punho & $9,24^{\circ}( \pm 1,17)$ & $0,8^{\circ}$ a $24,7^{\circ}$ \\
Extensão do Punho & $10,75^{\circ}( \pm 1,01)$ & $2,1^{\circ}$ a $24,7^{\circ}$ \\
Flexão MCF I Dedo & $13,71^{\circ}( \pm 1,44)$ & $2,1^{\circ}$ a $30,2^{\circ}$ \\
Extensão MCF I Dedo & $5,42^{\circ}( \pm 1,18)$ & $0,5^{\circ}$ a $15,7^{\circ}$ \\
Flexão IF I Dedo & $63,26^{\circ}( \pm 1,46)$ & $9^{\circ}$ a $105,2^{\circ}$ \\
Extensão IF I Dedo & $3,97^{\circ}( \pm 1,95)$ & - \\
Abdução CMC I Dedo & $47,54^{\circ}( \pm 1,30)$ & $36,7^{\circ}$ a $65,8^{\circ}$ \\
Flexão MCF II Dedo & $54,01^{\circ}( \pm 1,24)$ & $30,6^{\circ}$ a $76,7^{\circ}$ \\
Flexão IFP II Dedo & $75,29^{\circ}( \pm 1,47)$ & $33,1^{\circ}$ a $111,7^{\circ}$ \\
Flexão IFD II Dedo & $23,40^{\circ}( \pm 1,39)$ & $1,1^{\circ}$ a $54,9^{\circ}$ \\
Extensão IFD II Dedo & $20,09^{\circ}( \pm 1,27)$ & $1,2^{\circ}$ a $67,9^{\circ}$ \\
\hline
\end{tabular}




\section{Padrão postural na escrita}

Embora tenha ocorrido grande variação articular, foi possível observar um padrão postural durante a atividade de escrita manual, comum à população em questão e condizente com estudos prévios ${ }^{37,40,44,45}$, indicando um padrão articular minimamente estruturado entre os indivíduos que utilizam a preensão trípode dinâmica.

No presente estudo, a articulação do antebraço apresentou-se exclusivamente em pronação; os indivíduos permaneceram em abdução da articulação trapeziometacarpiana do I dedo, com abertura do primeiro espaço interdigital, flexão das articulações metacarpofalangeana e interfalangeana proximal do II dedo.

Observou-se que durante o desempenho da escrita manual houve grande variação da amplitude de movimento articular, sobretudo em metacarpofalangeanas e interfalangeanas, ao passo que articulações mais proximais como o punho apresentaram menor variabilidade.

\section{Discussão}

As articulações distais da mão apresentam maior ação e movimentação em padrões de preensão maduros, como a preensão trípode dinâmica, com predominância de pequenos movimentos de flexo-extensão dos dedos indicador e polegar e uso mínimo dos músculos proximais do membro superior e tronco ${ }^{16,25,37}$, indicando maior economia de energia e comodidade, se comparados com outros tipos de preensão considerados imaturos ou de transição $0^{1,13}$.

Em um dos primeiros estudos utilizando a análise de imagens para descrever modificações no padrão postural de crianças e adolescentes que utilizavam a preensão trípode dinâmica, Ziviani ${ }^{43}$ observa a presença de elementos comuns a indivíduos que utilizam este padrão de preensão: a manutenção do antebraço em pronação, desvio radial e extensão do punho, oponência do polegar e predomínio de movimentos de flexão das articulações interfalangeanas dos dedos I e II ${ }^{43}$.

Apesar do padrão observado entre os participantes deste estudo assemelhar-se àquele descrito por Ziviani ${ }^{43} \mathrm{e}$ demais autores ${ }^{6,12,20,25,37,44}$ foi possível observar modificações nas posições das articulações do punho e dedos: desvio ulnar e flexão do punho foram observados entre $22 \%$ e $16 \%$ dos participantes, respectivamente e a posição dos dedos demonstrou alterações entre flexão, extensão e hiperextensão das articulações metacarpofalangeana e interfalangeanas do I dedo e interfalangeana distal do II dedo.
Apesar de bem fundamentada por meio de numerosos estudos, a descrição da preensão trípode dinâmica muitas vezes exclui os movimentos de flexão do punho e hiperextensão das articulações interfalangeanas que comumente podem ser observados durante o desempenho de atividades de escrita.

É cada vez mais aceita entre a literatura especializada a variação entre flexão, extensão e até mesmo hiperextensão das articulações interfalangeanas proximais e distais ${ }^{12,40,41}$, conforme encontrado entre os sujeitos participantes deste estudo.

Summers ${ }^{40}$ observou prevalência de hiperextensão das articulações interfalangeanas em $71 \%$ de um grupo composto por 55 crianças, sendo a articulação interfalangeana do polegar aquela onde mais se observou hiperextensão. Em nosso estudo foi observado apenas um caso de hiperextensão da articulação interfalangeana do I dedo, com predominância da flexão desta entre a maioria dos indivíduos. Tal padrão vai de encontro com as descrições apresentadas por Edwards et al. ${ }^{12}$, onde a oponência do polegar em relação ao II dedo é considerada característica fundamental da preensão trípode dinâmica.

Os resultados confirmam que a hiperextensão do dedo indicador é uma característica prevalente na população estudada, com mais da metade dos indivíduos adotando esta posição. Segundo Elliot e Connolly ${ }^{13} \mathrm{o}$ dedo indicador e o polegar são os dígitos mais adaptados para exercer um controle motor fino e, por isso, devem-se manter em posturas facilitadas.

Selin (2003) aponta que uma preensão fácil seria aquela em que o primeiro e segundo dedos estão em estado relaxado, levemente flexionados ou com uma postura em linha reta. No entanto, os resultados obtidos em nosso estudo são similares aos de Ziviani ${ }^{43}$ e Summers ${ }^{40}$, que observam que esses dígitos são frequentemente vistos em posições heterodoxas, com as articulações interfalangeanas distais em hiperextensão, indicando pressão exagerada.

A hiperextensão é uma característica dominante nas preensões de crianças em idade escolar ${ }^{5,43}$, diminuindo com a idade, concomitante ao refinamento da preensão trípode dinâmica.

Os posicionamentos ou tipos de preensões incomuns são normalmente percebidos apenas quando o processo de escrita de um indivíduo é difícil, com a combinação de preensão anormal e/ou caligrafia desordenada e ilegível.

Em outras circunstancias, dificuldades e comprometimentos na atividade de escrita poderiam passar despercebidos ${ }^{3}$. Desta forma é importante ressaltar a importância do trabalho de orientação conjunta de educadores e terapeutas ocupacionais nos primeiros anos de vida escolar, 
para a detecção e correção de variáveis que possam levar a possíveis comprometimentos da atividade de escrita.

\section{Conclusão}

Embora atualmente a presença da escrita manual em tarefas cotidianas encontre-se reduzida pela ascensão de formas eletrônicas de produção textual, esta atividade mantém-se fundamenta para a progressão do indivíduo em ambientes educacionais. Desta forma, a avaliação e acompanhamento do desenvolvimento da escrita entre crianças, jovens e adultos que frequentem estes ambientes mostra-se uma importante área de atuação do terapeuta ocupacional.

O presente estudo objetivou a descrição, por meio da fotogrametria, de um padrão postural comum a indivíduos que utilizassem a preensão trípode dinâmica, preensão mais indicada por terapeutas e de maior prevalência entre a população.

Observou-se, apesar da grande heterogeneidade de posturas adotadas, uma estruturação de componentes articulares do membro superior, com pronação de antebraço, desvio radial, extensão de punho e flexão das articulações metacarpofalangeana dos dedos I e II e flexão da articulação interfalangeana proximal do II dedo.

A fotogrametria mostrou-se uma ferramenta confiável para análise da variação articular da mão durante a atividade de escrita. Tal dado mostra-se pertinente por indicar a possibilidade do uso desta ferramenta como meio para observar a evolução da escrita manual do sujeito, desde os primeiros anos de vida escolar até a chegada da vida adulta, permitindo obter conclusões sobre o real desenvolvimento das preensões e o padrão postural prevalente e funcional para a atividade manuscrita.

\section{REFERÊNCIAS}

1 Almeida PHT. Q. Análise eletromiográfica da escrita manual: estudo de dois padrões de preensão [Dissertação]. (Mestrado). São Carlos: Universidade Federal de São Carlos, Programa de Pós-Graduação em Terapia Ocupacional; 2012.

2 Amundson SJ. Prewriting and handwriting skills. In: Case-Smith J, editor. Occupational therapy for children. 5th ed. St Louis: Elsevier, Mosby; 2005. p.587-614.

3 Barnes KJ, et al. Perceptions regarding school-based occupational therapy for children with emotional disturbances. Am J Occup Ther. 2003;57(3):337-41.

4 Baur B, et al. Effects of modified pen grip and handwriting training on writer's cramp. Arch Phys Med Rehabil. 2009;9(5):867-75.

5 Benbow M. Principles and practice of teaching handwriting. In: Henderson A, Pehoski C, editors. Hand function in the child: foundations for remediation. 2nd ed. St. Louis: Mosby, Elsevier; 2006. p.321-44.

6 Bergmann KP. Incidence of atypical pencil grasps among nondysfunctional adults. Am J Occup Ther. 1990;44(8):736-40.

7 Bigaton DR, et al. Postura crânio-cervical em mulheres disfônicas. Rev Soc Bras Fonoaudiol. 2010;15:329-34.

8 Braz RG, Goes FPC, Carvalho GA. Confiabilidade e validade de medidas angulares por meio do software para avaliação postural. Fisioter Mov. 2008;21(3):117-26.

9 Chau T, et al. A novel instrument for quantifying grip activity during handwriting. Arch Phys Med Rehabil. 2006;87(11):1542-7.

10 Connelly V, Gee D, Walsh E. A comparison of keyboarded and handwritten compositions and the relationship with transcription speed. Br J Educ Psychol. 2007;77(Pt 2):479-92.

11 Couto HA. Ergonomia aplicada ao trabalho: o manual técnico da máquina humana. 2a ed. Belo Horizonte: Ergo; 1995.

12 Edwards SJ, Buckand DJ, Mccoy-Powlen JD. Grasps for Handwriting. In: Edwards SJ, Buckand DJ, et al., editors.. Developmental \& functional hand grasps. Thorofare: SLACK Incorporated; 2002. cap. 5, p.57-78.

13 Elliott JM, Connolly KJ. A classification of manipulative hand movements. Develop Med Child Neurol. 1984;26(3):283-96.

14 Falk TH, et al. Grip force variability and its effects on children's handwriting legibility, form, and strokes. J Biomech Eng. 2010;132(11):114504. doi: 10.1115/1.4002611.

15 Ferreira EAG, et al. Postural assessment software (PAS/ 
SAPO): validation and reliabiliy. Clinics. 2010;65:67581.

16 Ferriell BR, et al. Determining the effectiveness of pencil grips: an electromyographical analysis. Occup Ther Health Care. 2000;12(1):47-62.

17 Graup S, Santos SGD, Moro ARP. Estudo descritivo de alterações posturais sagitais da coluna lombar em escolares da rede federal de ensino de Florianópolis. Rev Bras Ortop. 2010;45:453-9.

18 Iunes D. et al. A influência postural do salto alto em mulheres adultas: análise por biofotogrametria computadorizada. Rev Bras Fisioter. 2008;12:441-6.

19 Iunes DH, et al. Confiabilidade intra e interexaminadores e repetibilidade da avaliação postural pela fotogrametria. Rev Bras Fisioter. 2005;9(3):327-4.

20 Koziatek SM, Powell NJ. Pencil grips, legibility, and speed of fourth-graders' writing in cursive. Am J Occup Ther. 2003;57(3):284-8.

21 Levine M, Brooks R, Shonkoff RP. A pediatric approach to learning disorders. New York: Wiley Medical; 1980.

22 Li-Tsang CW, et al. Handwriting characteristics among secondary students with and without physical disabilities: a study with a computerized tool. Res Develop Disabil. 2011;32(1):207-16.

23 Mathiowetz V, Bass-Haugen J. Assessing abilities and capacities: motor behavior. In: Radomski MV, Trombly Latham CA, editors. Occupational therapy for physical dysfunction. 6th ed. Baltimore: Lippincott Williams \& Wilkins; 2008. cap. 6, p.186-211.

24 Motta LJ, et al. Relação da postura cervical e oclusão dentária em crianças respiradoras orais. Rev CEFAC. 2009;11:298-304.

25 Naider-Steinhart S, Katz-Leurer M. Analysis of proximal and distal muscle activity during handwriting tasks. Am J Occup Ther. 2007;61(4):392-8.

26 Oliveira LM, Araujo PMP. Medidas de amplitude articular. In: Sociedade Brasileira de Terapia da Mão e do Membro Superior, editor. Recomendações para avaliação do membro superior. 2a ed. São Paulo: SBTM; 2005. p.43-54

27 Overvelde, A.; Hulstijn, W. Handwriting development in grade 2 and grade 3 primary school children with normal, at risk, or dysgraphic characteristics. Res Dev Disabil, Jan 242011.

28 Pardini AG. Anatomia funcional da mão. In: Freitas PP, editor. Reabilitação da mão. São Paulo: Atheneu; 2006. cap. 1, p.1-18.

29 Penso DE. Keyboard, graphic and handwriting skills: helping people with motor disabilities. London: Chapman and Hall; 1990.

30 Phillips JG, Ogeil RP, Muller F. Alcohol consumption and handwriting: a kinematic analysis. Hum Mov Sci. 2009;28(5):619-32.

31 Ribeiro AP, et al. Confiabilidade inter e intraexaminador da fotopodometria computadorizada e intra-examinador da fotopodoscopia. Rev Bras Fisioter. 2006;10(4):435-9.

32 Sacco I, et al. Confiabilidade da fotogrametria em relação a goniometria para avaliação postural de membros inferiores. Revista Brasileira de Fisioterapia, v. 11, p. 411-417, 2007.

33 Santos, M. et al. Análise postural fotogramétrica de crianças saudáveis de 7 a 10 anos: confiabilidade interexaminadores. Rev Bras Fisioter. 2009;13:350-5.

34 Sassoon R. The art and science of handwriting. Oxford: Intellect; 2004.

35 Scarone P, et al. Agraphia after awake surgery for brain tumor: new insights into the anatomo-functional network of writing. Surg Neurol. 2009;72(3):223-41; discussion 241.

36 Schneck CM. Comparison of pencil-grip patterns in first graders with good and poor writing skills. Am J Occup Ther. 1991;45(8):701-6.

37 Schneck CM, Henderson A. Descriptive analysis of the developmental progression of grip position for pencil and crayon control in nondysfunctional children. Am J Occup Ther. 1990;44(10):893-900.

38 Souza JA, et al. Biofotogrametria confiabilidade das medidas do protocolo do software para avaliação postural (SAPO). Rev Bras Cineantropometr Desempenho Hum. 2011;13:299-305.

39 Straker L, et al. Children have less variable postures and muscle activities when using new electronic information technology compared with old paper-based information technology. J Electromyogr Kinesiol. 2009;19(2):e13243.

40 Summers J. Joint laxity in the index finger and thumb and its relationship to pencil grasps used by children. Austr Occup Ther J. 2001;48(3):132-41.

41 Summers J, Catarro F. Assessment of handwriting speed and factors influencing written output of university students in examinations. Austr Occup Ther J. 2003;50(3):148-57. 
42 Van Drempt N, Mccluskey A, Lannin NA. Handwriting in healthy people aged 65 years and over. Austr Occup Ther J. 2011;58(4):276-86.

43 Ziviani J. Qualitative changes in dynamic tripod grip between seven and 14 years of age. Develop Med Child Neurol. 1983;25(6):778-82.

44 Ziviani J. Use of modern cursive handwriting and handwriting speed for children ages 7 to 14 years.
Percept Mot Skills. 1996;82(1):282.

45 Ziviani J, Elkins J. Effects of pencil grip on handwriting speed and legibility. Educ Rev. 1986;38:247-57.

46 Ziviani J, Wallen M. The development of graphomotor skills. In: Henderson A, Pehoski C, editors. Hand function in the child: fondations for remediation. 2nd ed. St Louis: Mosby Elsevier; 2006. cap. 11, p.21738 . 